\title{
Restriction Analysis of Transport Policy for Bridges Using the Trajectory Data
}

\author{
Zhenghua Hu, ${ }^{1,2}$ Jibiao Zhou $\mathbb{D}^{3,4}$ Shuichao Zhang, ${ }^{3}$ Songhan He, ${ }^{1}$ and Bo'an Yu${ }^{1}$ \\ ${ }^{1}$ School of Electronic and Information Engineering, Ningbo University of Technology, Fenghua Rd. No. 201, Ningbo 315211, \\ Zhejiang Province, China \\ ${ }^{2}$ College of Information Science \& Electronic Engineering, Zhejiang University, Zheda Rd. No. 38, Hangzhou 310027, \\ Zhejiang Province, China \\ ${ }^{3}$ School of Civil and Transportation Engineering, Ningbo University of Technology, Fenghua Rd. No. 201, Ningbo 315211, \\ Zhejiang Province, China \\ ${ }^{4}$ Department of Transportation Engineering, Tongji University, Caoan Rd. No. 4800, Shanghai 201804, China
}

Correspondence should be addressed to Jibiao Zhou; zhoujibiao@tongji.edu.cn

Received 24 August 2020; Revised 29 September 2020; Accepted 24 November 2020; Published 7 December 2020

Academic Editor: Xiaolei Ma

Copyright $(92020$ Zhenghua Hu et al. This is an open access article distributed under the Creative Commons Attribution License, which permits unrestricted use, distribution, and reproduction in any medium, provided the original work is properly cited.

\begin{abstract}
Roads are becoming increasingly congested with continuous rise in the number of vehicles. Restriction policies are selected to alleviate congestion in many cities. However, conclusions regarding the substantial effects of restriction policies have not been fully demonstrated. This study primarily aims to demonstrate whether traffic restrictions can control the driving habits of people to alleviate traffic pressure. Furthermore, this study investigates the effect on the traffic on the premise of a normalized restriction policy. Data were collected by bayonet systems in Ningbo. Results showed that vehicles restricted by the restriction policy only accounted for approximately $13 \%$. Most drivers bypass restricted roads to avoid restrictions. The method proposed can effectively amend the trajectory deviation caused by the inaccuracy from the bayonet. Based on the results, some suggestions about the policy of restrictions were discussed.
\end{abstract}

\section{Introduction}

With the continuous development of urbanization and the acceleration of motorization, traffic congestion has become a bottleneck in urban development. The congested roads in urban areas not only affect the traveling environment and physical health of residents but also seriously hinder the sustainable development of social economy and daily life [1-5]. According to the internationally accepted sayings, holding more than 1 million cars is a sign that a city has entered the "auto society." After the cities of Beijing, Shanghai, Chengdu, Guangzhou, Shenzhen, Tianjin, Hangzhou, and Suzhou, Ningbo has become the ninth city in China with more than one million cars. At the end of 2018, the total number of cars and drivers in Ningbo had reached 2.54 and 3.23 million, respectively. A total of 249,000 new cars and 213,000 new drivers were recorded in 2018 alone. The ownership of cars in the city of Ningbo continued to grow rapidly in 2019. Ningbo took only eight years to become a city with more than three million cars in 2019 from that with one million in 2011. The rapid growth in the total number of cars has caused congestion during rush hours in the core areas of Ningbo. The problem of "driving difficulty on the road" has become a livelihood issue that affects urban development and the lives of residents.

However, with the gradual improvement in road networks in the central area of Ningbo, the room for growth in infrastructure continues to decrease. Considering the local and international experience of development, the growth rate of transportation facilities has not sustained the demand from transportation.

In order to fundamentally solve the imbalances between supply and demand, it is necessary to treat the management of traffic demand as the fundamental means to solve transportation problems, fully tap the supply 
capacity of transportation facilities, and adjust the spatial-temporal distribution of traffic flows [6-8]. This finding has become a vital issue for the development of the transportation system and is also an essential direction for the strategies of transportation development in big cities in China.

Many researchers have comprehensively analyzed the causes of congestion and proposed corresponding strategies to solve the problem [9-12]. A popular method is to curb traffic demand by controlling the usage intensity of vehicles (purchase restriction policies [13], parking policies [14], schemes to encourage carpooling [15], etc.), thereby reducing the amount of traffic. Restrictions by license plate number are the most economical and effective way to reduce traffic pressure on the roads [16-20]. Traffic demand regulation through policies and economical means has been recently adopted in major cities. The city of Ningbo has also implemented traffic restrictions based on even- and odd-numbered license plates on bridges (Jiangxia and Ling Bridges) across the river in the core area of the city. However, some controversies over such strategies still exist. Some people believe that restrictions based on even- and odd-numbered license plates are unnecessary because these restrictions objectively break the original structure of urban traffic patterns, which causes traffic flow oscillation between the two bridges and other adjacent passages [21]. And, the effect of traffic demand management is slightly observed. However, others view that the restriction policy must be continuously implemented and further expanded to form restriction zones so as to achieve demand management in the core areas. Therefore, it is necessary to study this restriction policy, evaluate its effect and deficiencies in real-time, and propose suitable policies regarding traffic demand management in Ningbo.

\section{Literature Review}

2.1. Different Strategies on Traffic Restriction. The restriction policy has been implemented in some first-tier cities in China, such as Beijing, Shanghai, Guangzhou, and Hangzhou. Based on the rules, two main types of strategies are available: according to the tail number of the license plate $[22,23]$ and according to the exhaust emissions $[24,25]$. Among these strategies, traffic restriction according to plate number can be further divided into two subtypes, namely, restriction rules by even- and oddnumbered license plates and restriction of several $(N)$ numbers on each day of a week. Theoretically, the former can reduce traffic flow by $50 \%$ daily. In comparison, the latter can reduce the traffic flow by $10 \% \times N$. Based on the restricted time, the traffic restriction policies can be further divided into two subtypes: traffic restrictions during the special or nonspecial period. The former refer to restrictions during particular periods, such as the Olympic Games, Asian Games, or holidays, while the latter refer to restrictions during rush hours in the morning and evening on working days.
2.2. Evaluation of Restriction Policies. Researchers have obtained different evaluation results from different perspectives in studies. At present, the relatively acceptable evaluation conclusions are as follows:

(a) Policies of restrictions have changed the travel patterns of some residents; thus, these residents turn to public transportation, such as buses and subways [26]. However, these policies do not remarkably affect the travel of full-time personnel [27].

(b) Restricted rules have short-term effectiveness, but long-term restrictions will offset by various evasion behaviors [28, 29].

(c) Restriction policies can improve air quality to some extent [30]. Still, the effect is not evident [31, 32].

The advantages of implementing restriction policies are as follows.

(a) The cost of implementing restrictions is low

(b) In the early stage, the policies can reduce the traffic flow and alleviate traffic congestion $[25,33]$

(c) The policies control the exhaust emissions and improve the air quality to a certain extent $[34,35]$

The disadvantages of these policies are as follows [36, 37]:

(a) The traffic pressure can only be relieved in the short term and cannot fundamentally solve the problem of traffic congestion in urban areas

(b) These policies stimulate some people to purchase a second car to avoid traffic restrictions

The response from the public to restriction rules is various. Supportive and wait-and-see attitudes, as well as questioning voices, are observed [18, 20, 38]. Most citizens support the policy of traffic restriction. They believe that restriction rules can effectively stimulate citizens to take public transportation, such as buses and subways, thereby reducing exhaust emissions. Although the restriction policies have inconvenienced some car owners, they still support these restriction rules. However, some citizens believe that the restriction rules limit the legitimate rights and interests of citizens; and whether or not such restrictions provide corresponding compensation, the policy is considered a violation of private rights [39]. Many residents believe that with the gradual increase in motor vehicles, even with the adoption of restrictions rules, the willingness for people to drive out will not be curbed.

Overall, although restriction rules have limited the motorized vehicles out to a certain extent, long-term restrictions for travelers with complex needs may adopt evasion measures. Besides, the inconvenience caused by restriction may prompt the purchase of a second car, thereby inducing a new increase in vehicle ownership.

No researcher has implemented a detailed analysis from a quantitative perspective due to the different opinions on the restriction policy. Therefore, taking the restriction policy 
in Ningbo as an example, this study took the license plate data from bayonet systems to extract the trajectory of vehicles and then conducted a detailed evaluation of the restriction policy. The method used in this study refers to trajectory acquisition technology based on the license plate. Although the recognition of license plate has successful achievements at home and abroad, the conventional trajectory extraction technology cannot be directly applied in Ningbo, due to the particularity of the bayonets system (aging and inconsistency of the equipment or the inaccuracy of basic data, etc.). Therefore, introducing a series of data preprocessing and analysis methods is necessary.

\section{Data and Method}

3.1. Data Collection. Through real-time video monitoring in the Big Data Lab of Ningbo University of Technology, the license plate data are captured when cars pass through each bayonet. The data format is shown in Table 1 and Figure 1.

Furthermore, the information about traffic flow toward each direction can be calculated. Simultaneously, the erroneous data are eliminated, and the missing data are interpolated and fitted to obtain the complete trajectory of each vehicle. Thus, the flow, the degree of congestion (saturation), and the spatial distribution characteristics of traffic flow could be calculated.

3.2. Extraction Method of Traveling Trajectory. The original data must be preprocessed initially to remove errors because the recognition data of license plates captured by the bayonet system contain numerous gross errors due to objective reasons. Then, the unreasonable records are eliminated using positional and topological relationships between road network and bayonet stations. In the sample data, the set of bayonets passing by each vehicle for a single trip is queried after the grosses are removed, and the Dijkstra algorithm for the restricted area is called to calculate the path between two adjacent points. However, an absolute deviation is also found in the position of the bayonet; that is, the position of the bayonet and the road network data do not entirely match. Only the coverage tree of the travel path can be obtained. Finally, the actual trajectory is computed by finding the longest path in that covering tree.

3.3. Basic Data Analysis. The trajectory data from November 3, 2019, to November 30, 2019 (four entire weeks), were used to ensure the reliability and stability of statistical results. These data include more than 1.65 billion records with a volume of up to $100 \mathrm{G}$. Therefore, the statistical results are convincing. Finally, saturation and service level analyses for the bridges (Jiangxia and Ling Bridges) and the surrounding intersections were conducted using the aforementioned method. The daily average traffic volume of the road in the city of Ningbo reached 4.54 million vehicles, and the average flow on cross section was 162 vehicles/h. The changes in the traffic flow and saturation on the main roads are shown in Table 2.

\section{Case Study}

4.1. Basic Profiles. Ningbo is a subprovincial and a separate city under the jurisdiction of Zhejiang Province. It is an important port city approved by the State Council and is an economic center on the south side of the Yangtze River Delta. Ningbo is located in the middle of China's coastline, with the southern wing of the Yangtze River Delta and the Zhoushan Islands being a natural barrier in the east. The city of Ningbo is one of the eight major water systems in Zhejiang Province. The rivers include Yuyao, Fenghua, and Yongjiang Rivers. The Yuyao and Fenghua Rivers merge into the Yongjiang River at Sanjiangkou in the urban area. The Yongjiang River flows northeast and enters the East China Sea through Zhaobao Mountain. More than 10 bridges span over the central part of the Yuyao, Fenghua, and Yongjiang Rivers. Among these bridges, Ling and Jiangxia Bridges are the core bridges in Ningbo and are both the central artery of the city. The ends of the two bridges connect the Old Town of Haishu and Jiangdong Districts, as shown in Figure 2.

According to statistics, the number of private cars in Ningbo has reached 2.76 million as of May 2019, and the average daily number of trips by car in the central city reached 200,000. The restriction policy has been implemented in Ningbo for over 15 years (2004-2019). The effect of the policy indicates that despite traffic congestion alleviation or traveling structure adjustment, the actual role of the restriction rules is not as evident as expected. Considerable uncertainty is found in its actual effect.

Jiangxia and Ling Bridges are two east-west bridges located near Sanjiangkou in the center of Ningbo. They are both bridges that span over Fenghua River and connect Haishu with Yinzhou District. Both bridges are also an essential gateway to the new eastern town and the business district in the south. They are adjacent to the old district of Jiangdong and connect bustling Tianyi Square to the west. Jiangxia and Ling Bridges are two two-way and two-lane bridges. As essential traffic arteries in the center of Ningbo, Jiangxia and Ling Bridges bear the main traffic functions and traffic flow between Haishu and Yinzhou Districts in the downtown area of Ningbo.

Ling and Jiangxia Bridges can only accommodate limited traffic due to historical conditions. However, due to their essential location, these two bridges have the highest traffic flow daily among all main roads in Ningbo, which can accommodate nearly 30,000 cars per day. Therefore, the two bridges are always in a congested state before the restriction policy was carried out, and the rush hours lasted for more than four hours a day.

4.2. Analysis of Trips under Traffic Restrictions. The management department should take economic or administrative measures, such as restriction rule, not only to control the travel demand and guide people to switch to public transportation (such as buses and subways), but also to reduce the actual traffic flow of road networks. This will undoubtedly optimize the relationship between traffic demand and supply, ease the traffic pressure on the road, maintain 
TABLE 1: Data description of license plate captured by bayonet.

\begin{tabular}{lcc}
\hline Name & Definition & Description \\
\hline $\begin{array}{l}\text { DeviceID } \\
\text { SnapShottime }\end{array}$ & $\begin{array}{c}\text { Number of each device } \\
\text { Shooting time } \\
\text { LaneNo }\end{array}$ & An 18-digit number that uniquely identifies a bayonet \\
CarType & Sequence of the lane & Time of each shot \\
PlateNum & Vehicle type & Sequence of the lane \\
& Plate number & 1 for car, 2 for truck, 3 for bus, and 4 for minibus \\
Number of the recognized license plate
\end{tabular}



- Survey site

Figure 1: Road network and bayonet distribution on the road.

TABLe 2: Statistics of traffic flow on restricted bridges and intersections nearby.

\begin{tabular}{|c|c|c|c|c|c|c|c|c|c|c|c|c|c|c|}
\hline \multirow{3}{*}{ Statistical section } & \multicolumn{14}{|c|}{ Statistical period } \\
\hline & \multicolumn{2}{|c|}{$7: 00-8: 00$} & \multicolumn{2}{|c|}{$8: 00-9: 00$} & \multicolumn{2}{|c|}{$\begin{array}{c}\text { 10:00-11: } \\
00\end{array}$} & \multicolumn{2}{|c|}{$\begin{array}{c}\text { 13:00-14: } \\
00\end{array}$} & \multicolumn{2}{|c|}{$\begin{array}{c}\text { 17:00-18: } \\
00\end{array}$} & \multicolumn{2}{|c|}{$\begin{array}{c}\text { 18:00-19: } \\
00\end{array}$} & \multicolumn{2}{|c|}{$\begin{array}{c}20: 00-21: \\
00\end{array}$} \\
\hline & $\mathrm{F}$ & S & $\mathrm{F}$ & S & $\mathrm{F}$ & S & $\mathrm{F}$ & S & $\mathrm{F}$ & S & $\mathrm{F}$ & S & $\mathrm{F}$ & S \\
\hline Jiangxia Bri & 1013 & 0.96 & 820 & 0.78 & 795 & 0.76 & 829 & 0.79 & 1078 & 1.03 & 989 & 0.94 & 1308 & 1.25 \\
\hline Ling Bridge & 739 & 0.70 & 623 & 0.59 & 609 & 0.58 & 628 & 0.60 & 778 & 0.74 & 772 & 0.74 & 903 & 0.86 \\
\hline Intersections around Jiangx & 1103 & 1.05 & 1108 & 1.06 & 1119 & 1.07 & 1197 & 1.14 & 1327 & 1.26 & 1235 & 1.18 & 1483 & 1.41 \\
\hline Intersections around Ling Bridge & 749 & 0.71 & 704 & 0.67 & 694 & 0.66 & 700 & 0.67 & 838 & 0.80 & 746 & 0.71 & 772 & 0.74 \\
\hline
\end{tabular}

F: flow (vehicles/hour) and S: saturation.

relatively smooth state of traffic flow, and improve the traveling environment. Vehicles running at a relatively smooth speed on the road can effectively reduce vehicle exhaust emissions, thereby further improving the air quality of the city.

4.2.1. Analysis of the Effect of Restriction Rules. This study uses the data of license plate from bayonet system in November 2019 to extract trajectory from vehicles and analyze whether the restriction policy has achieved the intended purpose of restricting traffic in Ningbo.

According to the restriction rules in Ningbo, the trajectory of vehicles that pass through the restricted bridges on the first day was calculated the next day. Then, these trajectories are used to verify whether the restriction policy effectively restrained people's habit of driving out and shifted the traveling mode to public transportation.

The statistical data show that the vehicle restriction policy in Ningbo is not as effective as expected. The number of vehicles that are restricted only accounts for approximately $13 \%$. That is, only $13 \%$ of vehicle owners give up driving out when facing the policy and select to travel by bus, subway, or nonmotorized vehicles. The restriction policy has changed and adjusted people's traveling mode to some extent. The policy has also alleviated the city's traffic pressure to a certain extent. Therefore, the restriction policy has played a role in the city of Ningbo, but the effect is not evident. Most car owners do not change their traveling modes but respond to restriction rules by bypassing corresponding roads.

4.2.2. Analysis of the Effect of Limiting Traffic. Jiangxia and Ling Bridges have become the most critical bridges to the new town in the east due to their unique locations. Long before the implementation of the traffic restriction, the two bridges were well known to the public. The average traffic flow of the two bridges on working days was computed to 


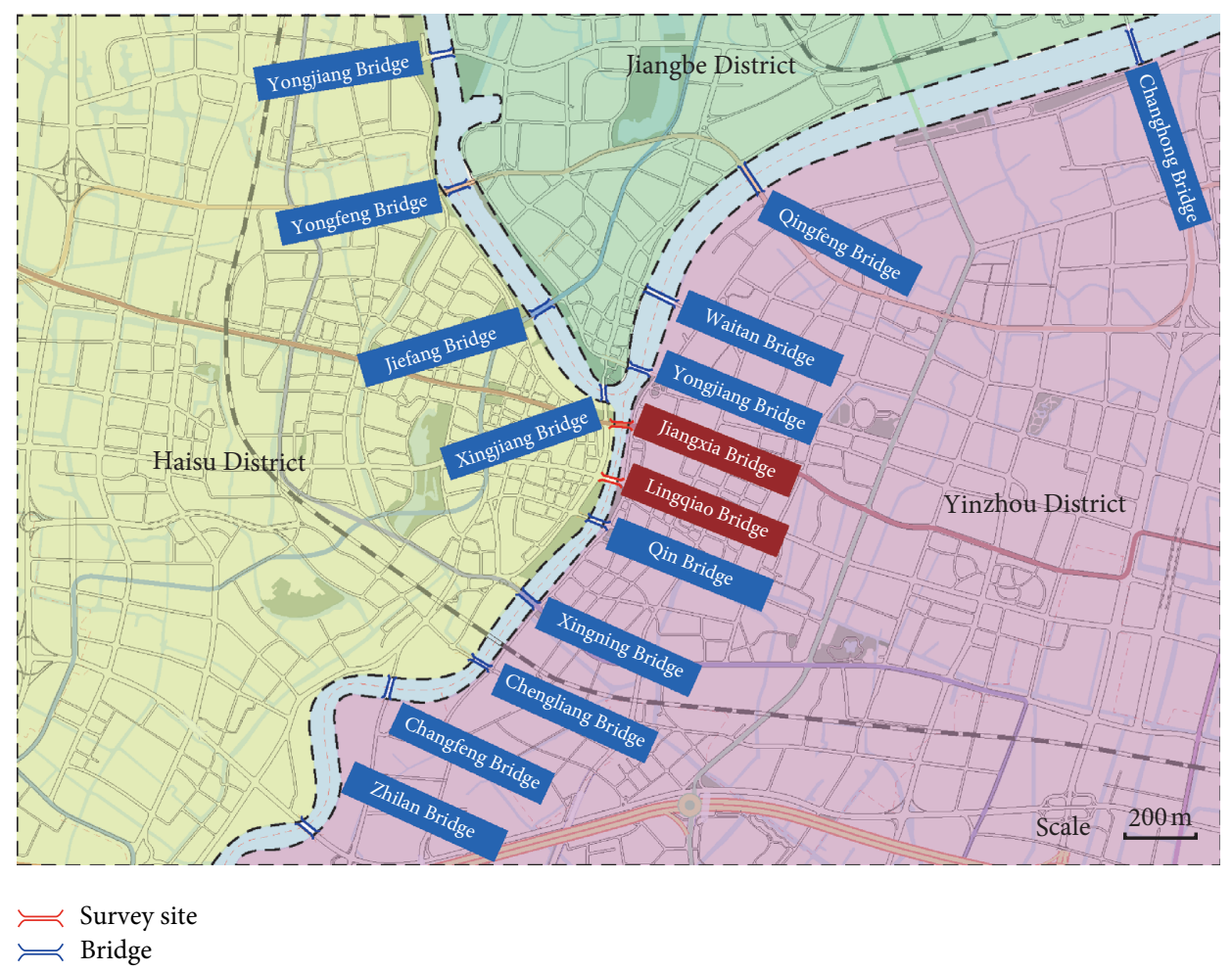

Figure 2: Overview of the research area.

verify the effect of restriction policies on the bridge, as shown in Figure 3.

The chart shows that, during the restricted time, the traffic volume on Jiangxia and Ling Bridges did not show a significant peak and remained within a relatively stable range. The effect of traffic control on the corresponding road is evident. As the most important arterial road in the city center, the restriction policy has reduced the overall traffic volume on Jiangxia and Ling Bridges by $20 \%-30 \%$. The restriction policy also reduced the traffic volume of the relevant roads and increased the average running speed. Thus, the roads were in a state of "basically unobstructed." However, according to the regulations of restrictions based on even- and odd-numbered license plates, nearly half of the traffic flow should be restricted from passing through the restricted roads in theory, but the ideal state was never reached. This finding may be due to the fact that the reduced traffic volume on the restricted roads has attracted traffic elsewhere or that some families have purchased a second car to deal with the restriction policy.

Outside the restricted period, the traffic volume of the restricted roads may be slightly higher than that during the restricted period. Significantly, the traffic volume of the restricted roads shows a maximum value within half an hour before and after the restricted period. This finding indicates that many travelers have changed the original travel time and passed the restricted road during nonrestricted periods (before or after the restricted time). Many drivers even pass through the restricted roads in the restricted time.

The traffic restriction has played a role in limiting the traffic volume for the two bridges, and the effect on traffic is evident. This finding also shows that the restriction policy diverts traffic from rush hours to flat hours. The policy improves the passability of the road and alleviates the current situation of traffic congestion in the morning and evening peaks.

4.2.3. Analysis of the Shunt Effect. Furthermore, the detour trajectories (trajectory of vehicles with odd number on even days and trajectory of vehicles with even number on odd days) during workdays are computed within four complete weeks, as shown in Figure 4.

The statistical data show that, due to the restriction policy, vehicles that originally traveled from Jiangxia and Ling Bridges were detoured from other bridges nearby. Moreover, the detour routes were mainly concentrated in Jiefang and Bund Bridges that locate at the north side of Jiangxia Bridge, Qin and Xingning Bridges that locate at the south side of the Ling Bridge, and the Zhilan Bridge that locates at the southwest of the city.

To the north of Jiangxia Bridge, nine detours comprising six bridges, including the Bund, Xinjiang, Yongjiang, and Jiefang Bridges, are available for vehicles to bypass. Among these bridges, the Jiefang and Bund Bridges have become the most welcomed detour routes for drivers, accounting for $12 \%$ of all detoured traffic because they are the most convenient alternative passages. The Xinjiang and Yongjiang Bridges are the closest to the Jiangxia Bridge; thus, many vehicles (approximately $8 \%$ ) choose to pass through the two bridges. The reason is mainly that some drivers forget the regulations of that day when traveling. Moreover, many 


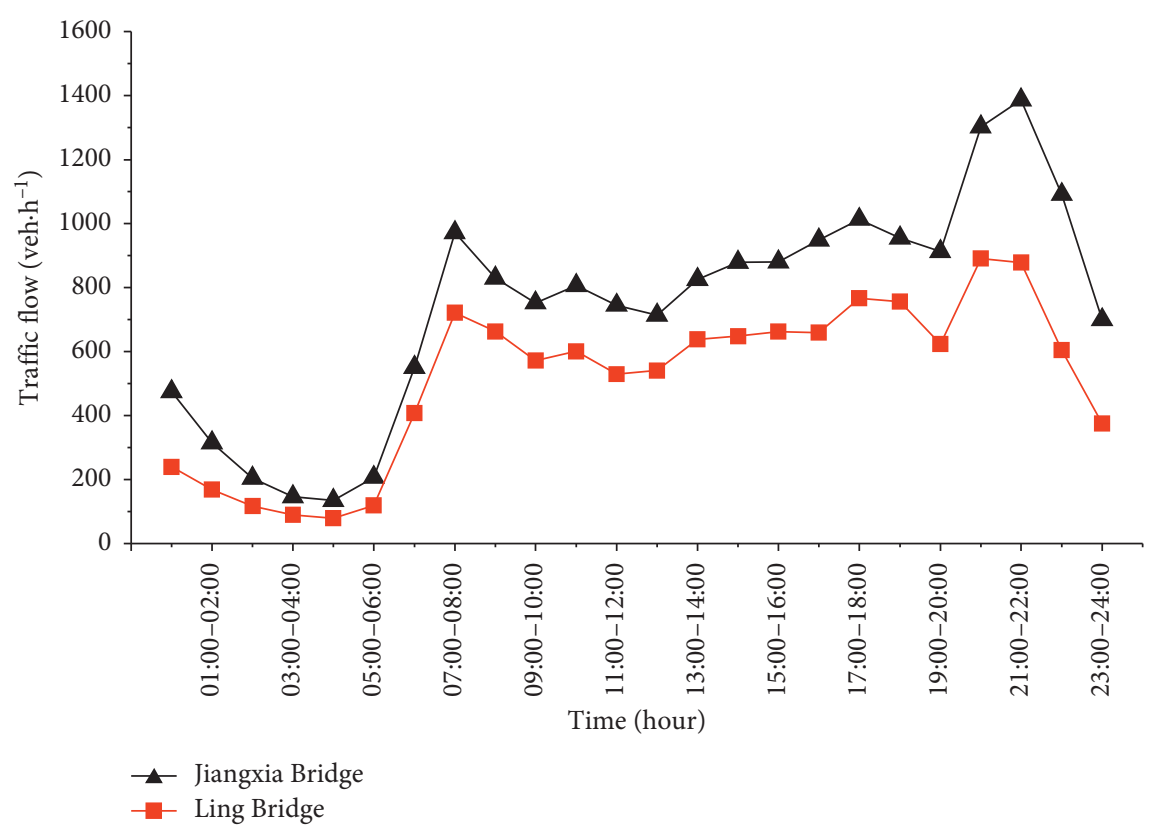

FIgURE 3: Daily average flow of the two restricted bridges.

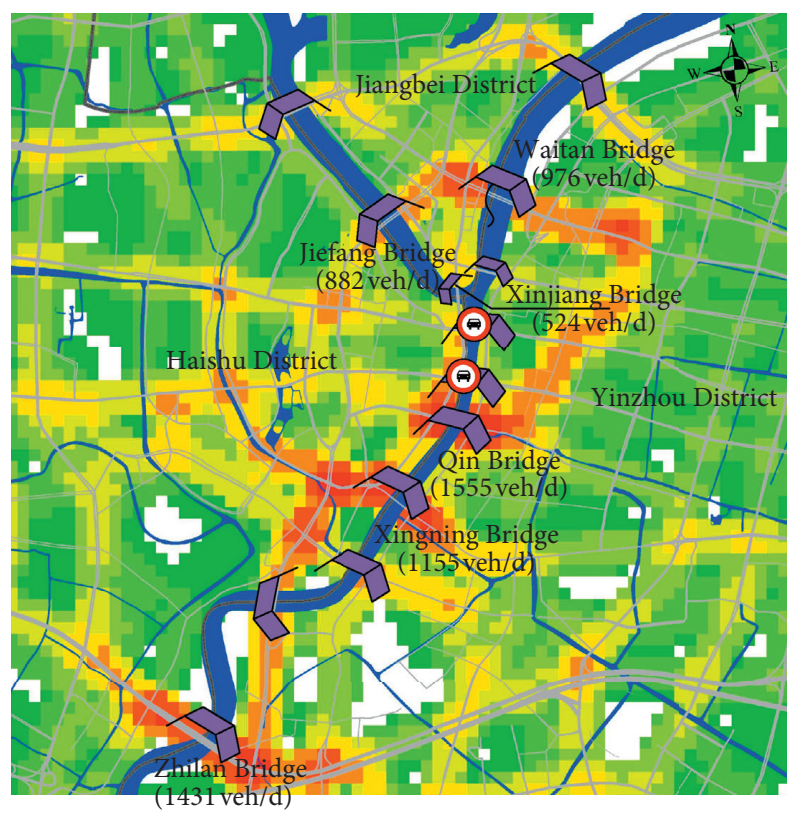

(a)

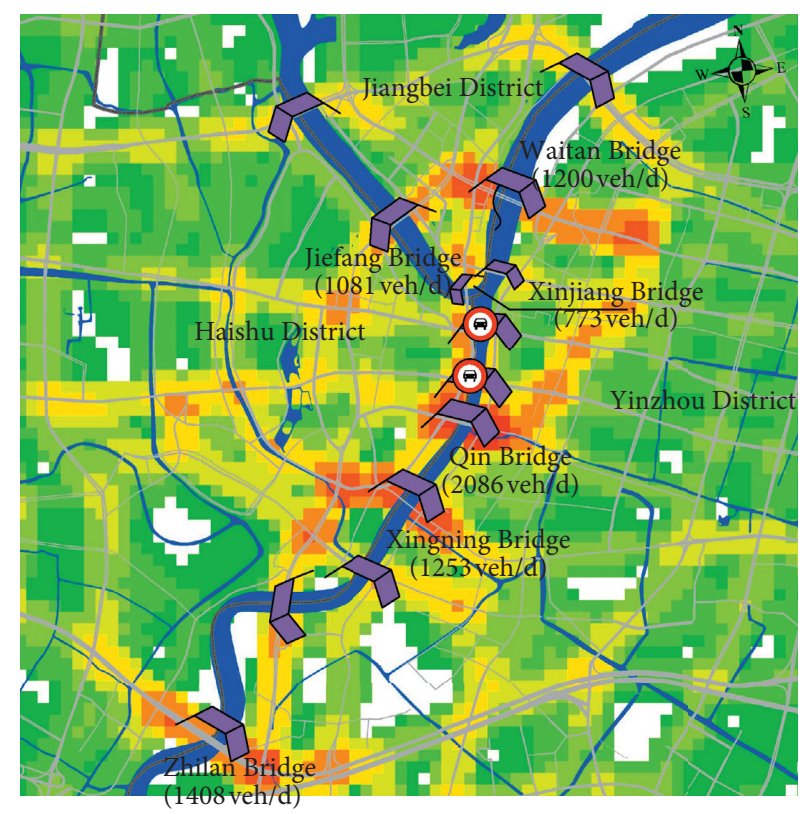

(b)

FIGURE 4: Heat maps of the detours. (a) Trajectory of vehicles with odd number on even days. (b) Trajectory of vehicles with even number on odd days.

foreign drivers do not know the local traffic restrictions in Ningbo; thus, these drivers change their paths temporarily after seeing a warning sign in front of the bridge. Other detour routes have either certain inconveniences or poor road conditions, and relatively few detour vehicles are available.

The detour route to the south mainly includes the Qin and Xingning Bridges. Although the Qin Bridge is geographically closest to Ling Bridge, this bridge has a long history and has limited traffic capacity. Therefore, the traffic conditions of the bridge are relatively bad. Most drivers detour from Xingning Bridge, which is not far from the Qin Bridge. Therefore, the Qin and Xingning Bridges have become the most crucial bypass bridges in the south, bearing $15 \%$ and $18 \%$ of the detoured traffic, respectively. The statistical results also show that the South Ring Road, which is the farthest from the restricted bridge, is a significant detour. The reason is that this road combines viaduct in the 
mainline and auxiliary on the ground, so the overall traffic condition is good. Moreover, the west side of the road is connected to the airport viaduct; therefore, the road has a considerable traffic capacity. Hence, a large proportion $(16 \%)$ of drivers is willing to sacrifice the running length to ensure the punctuality of the travel.

\subsubsection{Analysis of the Impact of Restriction Rules on Traffic} Flow. Although the restriction policy does not effectively curb private cars running on the roads, this policy has controlled the traffic flow on restricted roads to a certain extent, exhibiting a diversion effect on the traffic flow. This finding also has a specific impact on the roads around the restricted sections. This study used the license plate data captured by the bayonet to extract the travel trajectory and calculate the changes in indicators on the detours around the two restricted bridges (Jiangxia and Ling Bridges). The impact of the restriction policy on unrestricted roads is then studied. The results are shown in Table 3.

Table 3 shows that the restriction policy affected the flow of bypass-roads around the restricted bridges. The policy has caused many travelers to detour from other bridges around restricted ones. Thus, the traffic flow on the bypass-roads was significantly improved, especially for Qingfeng and Xinjiang Bridges. However, these roads have not experienced explosive growth, and the traffic flow on the entire urban road network can be maintained in a smooth state. This finding is also consistent with the conclusions from the previous analysis. The restricted vehicles have experienced additional miles due to detours. The total mileage has increased for each trip, but the increment is within an acceptable range. The average length of the detour indicates that the distance due to the restriction accounts for $10 \%$ of the total mileage. The average driving speed denotes that as the traffic volume on the relevant road increases, the number of vehicles passing through increases and the average speed of the corresponding road decreases due to the increased traffic flow. These findings are consistent with the actual situation.

Based on the length of a single trip and the required travel time, the delayed time is further estimated, as shown in Figure 5. The figure indicates that although the traffic flow on the road and the total length of the path have increased to varying degrees, no explosive growth in the final travel time was observed. The time with and without detour is almost equal. For many detours, the floating range of the delay is within the acceptable level. Because of the restriction policy, the travel period has been extended. Hence, the distribution of travel flow per hour has become more balanced. Significantly, the proportion of trips in the morning and evening rush hours has reduced. Simultaneously, the proportion of trips before morning rush and after evening rush has increased. This indicates that the restriction policy effectively realizes the regulation of traveling time and optimizes the supply and demand relationship of traffic during rush hours. Therefore, increasing the travel distance in exchange for an improved driving environment is relatively unacceptable. As a whole, if a comfortable driving environment is ensured, then most travelers are willing to travel from detours.

4.2.5. Scenario Simulation. We analyzed the different effects of restriction policy based on even- and odd-numbered license plates upon cross-river passages in Ningbo. Based on the existing travel trajectory, we explore the following three traffic scenarios with different restriction policies:

Scenario I: the status of all cross-river passages remains unchanged; that is, traffic restriction policy is continued to be carried out on Jiangxia and Ling Bridges

Scenario II: all cross-river passages adopt traffic restriction policy based on even- and odd-numbered license plates

Scenario III: all cross-river passages do not adopt traffic restriction policy based on even- and odd-numbered license plates

(1) Scenario I. The traveling trajectories are counted using extraction method proposed in this paper. The traffic flow of the two restricted bridges is about $1137 \mathrm{veh} / \mathrm{h}$, the saturation is 1.03 , the number of vehicles that run across the river is 14219 , and the number of detouring vehicles is 8866 .

(2) Scenario II. Combined with the optimization of the traffic structure and development trend, a low-carbon traveling structure is proposed, and all cross-river passages are restricted by even- and odd-tail numbers. The traffic flow of the two restricted bridges is $1006 \mathrm{veh} / \mathrm{h}$, the saturation is 0.91 , the number of vehicles that run across the river is 12578 , and the number of detouring vehicles is 6805 .

(3) Scenario III. Since all cross-river passages do not adopt restriction policy, this suppresses the tendency of citizens to buy a second car to a certain extent. In the beginning, the traffic volume of the cross-river passages that cancel the restriction rules will increase, but as time goes on, the traffic will gradually and automatically tend to a balanced state. The traffic flow of the two restricted bridges is $1847 \mathrm{veh} / \mathrm{h}$, the saturation is 1.67 , the number of vehicles that run across the river is 23088, and the number of detouring vehicles is 5353 .

Table 4 summarizes the information (such as traffic volume) on Jiangxia and Ling Bridges under three different traffic control methods.

On the whole, due to the increasing travel demand from residents, the growth trend of car ownership is obvious. In Scenario I, if the existing restriction policy remained unchanged, then most drivers would choose to detour to avoid restrictions that are imposed by the policy. In Scenario II, if all cross-river passages adopt the restriction policy, the proportion of public transport trips will undoubtedly increase, and saturation of roads will decrease. But there would be a tendency in saturation to increase year by year. Due to wealth of household and other factors, the number of private cars in a family may be more than one, which would cause more serious congestion. Within a few years, the traffic volume of the two bridges will return to the 
TABLE 3: Indicators of traffic flow on detours before and after adopting the restriction rule.

\begin{tabular}{|c|c|c|c|c|c|c|}
\hline \multirow[t]{2}{*}{ Bridges } & \multicolumn{2}{|c|}{$\begin{array}{c}\text { Average flow on detours } \\
(\mathrm{v} / \mathrm{h})\end{array}$} & \multicolumn{2}{|c|}{$\begin{array}{l}\text { The average length of the } \\
\text { detours }(\mathrm{km})\end{array}$} & \multicolumn{2}{|c|}{ Average speed $(\mathrm{km} / \mathrm{h})$} \\
\hline & Before & After & Before & After & Before & After \\
\hline QFB-YFB & 2978 & 3091 & 12.77 & 13.13 & 25.6 & 25.2 \\
\hline QFB-JFB & 475 & 487 & 12.06 & 14.21 & 17.9 & 22.2 \\
\hline QFB-XJB & 644 & 759 & 11.65 & 11.94 & 23.1 & 23.9 \\
\hline WTB-YFB & 1996 & 2115 & 12.35 & 12.58 & 20.9 & 20.7 \\
\hline WTB-JFB & 2386 & 2656 & 9.19 & 10.18 & 18.4 & 19.3 \\
\hline WTB-XJB & 392 & 408 & 14.19 & 14.14 & 11.1 & 15.7 \\
\hline YJB-YFB & 663 & 665 & 9.84 & 9.86 & 19.5 & 19.5 \\
\hline YJB-JFB & 1075 & 1084 & 8.05 & 7.86 & 19.2 & 19.2 \\
\hline YJB-XJB & 869 & 909 & 8.52 & 8.21 & 17.5 & 18.9 \\
\hline Qin Bridge & 10066 & 11290 & 7.98 & 8.05 & 17.3 & 17.5 \\
\hline $\mathrm{XNB}$ & 7140 & 7630 & 10.39 & 10.49 & 19.8 & 19 \\
\hline CFB & 11395 & 11637 & 11.26 & 11.9 & 20.3 & 19.3 \\
\hline CLB & 10706 & 10986 & 9.46 & 11.17 & 18 & 17.2 \\
\hline Zhilan Bridge & 11115 & 11556 & 12.12 & 13.7 & 26.8 & 25.2 \\
\hline
\end{tabular}

QFB is short for Qingfeng Bridge, YFB is short for Yongfeng Bridge, JFB is short for Jiefang Bridge, XJB is short for Xinjiang Bridge, WTB is short for Waitan Bridge, YJB is short for Yongjiang Bridge, XNB is short for Xingning Bridge, CFB is short for Changfeng Bridge, and CLB is short for Chenglang Bridge.

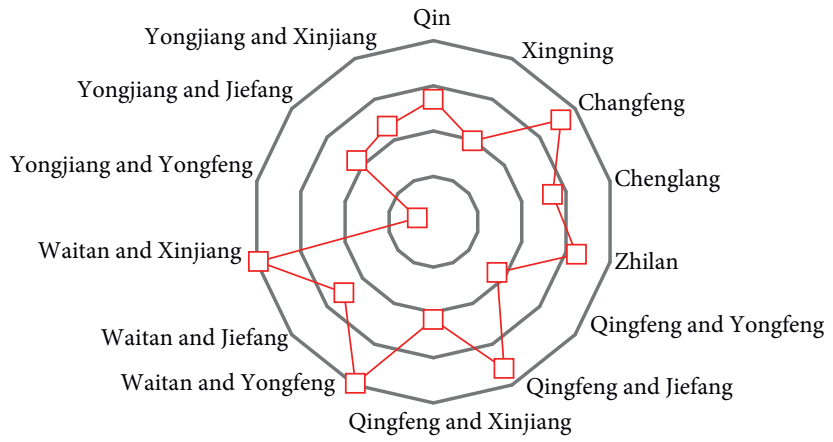

FIGURE 5: Delays from driving around the detoured bridges.

TABLE 4: Traffic information on Jiangxia and Ling Bridges under three different scenarios.

\begin{tabular}{lcccc}
\hline Scenarios & Traffic flow & Saturation & Number of vehicles run across the river & Number of detouring vehicles \\
\hline Scenario I & 1137 & 1.03 & 14219 & 8866 \\
Scenario II & 1006 & 0.91 & 12578 & 6805 \\
Scenario III & 1847 & 1.67 & 23088 & 5353 \\
\hline
\end{tabular}

level before the restriction policy was carried out. In Scenario III, the restriction policy was not carried out on all passages across the river. Although the traffic volume on the previously restricted bridges has increased, due to the self-adaptability, the traffic volume of either bridge will gradually stabilize without sharp fluctuations. In contrast, Scenario III is more effective in alleviating traffic congestion in Ningbo.

4.3. Evaluation of Restricted Policies. From the previous analysis, the conclusions are drawn as follows:

(a) Traffic restriction policy can effectively control the traffic flow on restricted roads, thereby improving the speed and substantially reducing the traffic pressure on restricted roads. Meanwhile, the restriction policy can restrain residents from using private cars to a certain extent and guide them to shift their travel ways to public transportation, such as buses or subways. This can alleviate exhaust pollution on the road and improve air quality in cities. The government has achieved the effect of guiding users' options of travel modes by implementing restrictions rules.

(b) In the face of restrictions, only a small number of citizens travel by bus or subway during the restricted period, and most citizens still use private cars to travel. Among these citizens, some travel out of the restricted period to avoid restriction rules. An increasing number of citizens will opt to detour roads around the restricted roads, thereby increasing the traffic pressure on the surrounding detours. The restriction policy plays a role in diverting traffic on restricted roads. 
(c) In the long run, high-income people will purchase a second car to cope with the restriction policy. The increment in the number of vehicles will not fundamentally improve the situation of congestion on the road. Therefore, the long-term implementation of the restriction policy cannot control the overall traffic volume, and the effect of the restriction policy will be significantly reduced.

Overall, the policy of traffic restriction has limited people from using cars to a certain extent. In particular, this policy significantly affects traffic on restricted roads, ensures driving efficiency, and improves the traffic order. Restriction policy has reasonably promoted people's dependence on public transportation. However, these measures have not achieved the expected effect. Most car owners either travel during the nonrestriction period or bypass the restricted road. The occupancy rate of vehicles on the detours has significantly increased during the restriction period. In other words, traffic restriction has only played a role in diverting traffic on restricted roads.

\section{Conclusion}

We believe that the traffic flow on Ling Bridge and Jiangxia Bridge has experienced a historical decline, indicating that the restriction policy controls the traffic flow on relevant roads to a certain extent. Therefore, the traffic restriction policy has played a role in regulating traffic demand. However, most drivers circumvent restrictions by bypassing from either side of restricted bridges. If the traffic restriction policy was canceled, the traffic flow of Jiangxia Bridge and Ling Bridge, as well as the nearby roads, would inevitably rebound. However, due to the limited capacity itself, the traffic flow would be self-adaptive and return to a stable state. In particular, we believe that the traffic problem in Ningbo is the problem of commuter traffic. The traffic pressure during rush hours is relatively small (in core areas, trips by car in the four rush hours in the morning and evening account for $69.7 \%$ of the entire day and do not exceed $6 \%$ during flat hours). If the restriction policy was canceled, then no unprecedented pressure would be found from traffic.

From the perspective of the traffic control functions, the effect of restriction policy includes traffic flow regulation on certain roads. This effect is limited for the adjustment of regional traffic demand and may still be affected by the overall capacity of roads. The separate effect of restriction policy is small. So the restriction rules should be complemented by other transportation strategies (such as increasing parking fees and reducing public transportation fees) to improve their effects.

Further research on the reoptimization of road spaces to realize the continuity of motor vehicles, public transportation, nonmotorized vehicles, and sidewalks will be conducted. The focus is on improving the quality of public transportation, nonmotorized vehicles, walking, and maximizing the role of slow-moving traffic. Relevant departments should gradually increase the cost of cars and promote the transformation from short-distance travel by car to nonmotorized vehicles or walking. Meanwhile, additional crowdsourced data, such as trajectory and mobile phone signaling data, must be collected to further verify the effectiveness of the proposed method, especially in cities (such as Wuhan and Nanjing) with similar terrain in Ningbo where the entire city is divided by rivers or mountains into different regions.

\section{Data Availability}

The codes that support the findings of this study are available at https://figshare.com/articles/software/Restric tion_Analysis_of_Transport_Policy_for_Bridges_Using_ the_Trajectory_Data/11783649.

\section{Conflicts of Interest}

The authors declare that they have no conflicts of interest.

\section{Acknowledgments}

This research was funded by Natural Science Foundation of Zhejiang Province (nos. LQ18D010008 and LQ19E080003), National Natural Science Foundation of China (Grant no. 52002282), Philosophy and Social Science Foundation of Zhejiang Province (no. 21NDJC163YB), Natural Science Foundation of Ningbo (nos. 2018A610132 and 2019A610044), Philosophy and Social Science Foundation of Ningbo (no. G20-ZX37), and Scientific Research Fund of Zhejiang Provincial Education Department (Y201736984).

\section{References}

[1] F. Chen, M. Song, and X. Ma, "Investigation on the injury severity of drivers in rear-end collisions between cars using a random parameters bivariate ordered probit model," International Journal of Environmental Research and Public Health, vol. 16, no. 14, Article ID 2632, 2019.

[2] F. Chen and S. Chen, "Injury severities of truck drivers in single- and multi-vehicle accidents on rural highways," $A c$ cident Analysis \& Prevention, vol. 43, no. 5, pp. 1677-1688, 2011.

[3] F. Chen, J. Wang, and Y. Deng, "Road safety risk evaluation by means of improved entropy TOPSIS-RSR," Safety Science, vol. 79, pp. 39-54, 2015.

[4] C. Ma, W. Hao, W. Xiang, and W. Yan, "The impact of aggressive driving behavior on driver-injury severity at highway-rail grade crossings accidents," Journal of Advanced Transportation, vol. 2018, Article ID 9841498, 10 pages, 2018.

[5] Y. Guo, T. Sayed, and M. Essa, "Real-time conflict-based Bayesian tobit models for safety evaluation of signalized intersections," Accident Analysis \& Prevention, vol. 144, Article ID 105660, 2020.

[6] F. Chen, S. Chen, and X. Ma, "Analysis of hourly crash likelihood using unbalanced panel data mixed logit model and real-time driving environmental big data," Journal of Safety Research, vol. 65, pp. 153-159, 2018.

[7] Y. Guo, M. Essa, T. Sayed, M. M. Haque, and S. Washington, "A comparison between simulated and field-measured conflicts for safety assessment of signalized intersections in 
Australia," Transportation Research Part C: Emerging Technologies, vol. 101, pp. 96-110, 2019.

[8] Y. Guo, Z. Li, P. Liu, and Y. Wu, "Modeling correlation and heterogeneity in crash rates by collision types using full Bayesian random parameters multivariate tobit model," Accident Analysis \& Prevention, vol. 128, pp. 164-174, 2019.

[9] X. Li, W. Wang, C. Xu, Z. Li, and B. Wang, "Multi-objective optimization of urban bus network using cumulative prospect theory," Journal of Systems Science and Complexity, vol. 28, no. 3, pp. 661-678, 2015.

[10] X. Li, J. Tang, X. Hu, and W. Wang, "Assessing intercity multimodal choice behavior in a touristy city: a factor analysis," Journal of Transport Geography, vol. 86, Article ID 102776, 2020.

[11] Y. Guo, T. Sayed, L. Zheng, and M. Essa, "An extreme value theory based approach for calibration of microsimulation models for safety analysis," Simulation Modelling Practice and Theory, vol. 106, Article ID 102172, 2021.

[12] Y. Guo, T. Sayed, and L. Zheng, "A hierarchical Bayesian peak over threshold approach for conflict-based before-after safety evaluation of leading pedestrian intervals," Accident Analysis \& Prevention, vol. 147, Article ID 105772, 2020.

[13] F. Liu, F. Zhao, Z. Liu, and H. Hao, "The impact of purchase restriction policy on car ownership in China's four major cities," Journal of Advanced Transportation, vol. 2020, no. 5, 14 pages, Article ID 7454307, 2020.

[14] A. Ibeas, L. dell'Olio, and J. L. Moura, "Parking behavior and policy," Journal of Advanced Transportation, vol. 20182 pages, Article ID 1075946, 2018.

[15] D. Ding and B. Shuai, "A traffic restriction scheme for enhancing carpooling," Discrete Dynamics in Nature and Society, vol. 2017, Article ID 9626938, 9 pages, 2017.

[16] J. A. Acero, A. Simon, A. Padro, and O. S. Coloma, "Impact of local urban design and traffic restrictions on air quality in a medium-sized town," Environmental Technology, vol. 33, no. 21, pp. 2467-2477, 2012.

[17] X. Liu, "Analysis of the policy of "peak shifting the limit line" in Hangzhou," Advances in Social Sciences, vol. 8, no. 4, pp. 556-559, 2019.

[18] S. Lucrezi, M. Saayman, and P. Van der Merwe, "Impact of off-road vehicles (ORVs) on ghost crabs of sandy beaches with traffic restrictions: a case study of Sodwana Bay, South Africa," Environmental Management, vol. 53, no. 3, pp. 520-533, 2014.

[19] K. Ostrowski and M. Tracz, "Availability and reliability of a signalised lane," Transportmetrica B: Transport Dynamics, vol. 7, no. 1, pp. 1044-1061, 2019.

[20] A. Szarata, K. Nosal, U. Duda-Wiertel, and L. Franek, "The impact of the car restrictions implemented in the city centre on the public space quality," Transportation Research Procedia, vol. 27, pp. 752-759, 2017.

[21] X. Cheng, K. Huang, L. Qu, T. Zhang, and L. Li, "Effects of vehicle restriction policies on urban travel demand change from a built environment perspective," Journal of Advanced Transportation, vol. 2020, no. 3, 13 pages, Article ID 9848095, 2020.

[22] X. Dong, The Impact of Travel Habits for Private Car on Travel Choice under the One-Day-a-Week Limiting Policy in Beijing, Beijing Jiaotong University, Beijing, China, 2019.

[23] Z. Sun, The Influence of Beijing Vehicle Restrictions on the Travel Mode Choice of Residents-an Empirical Study, Beijing Jiaotong University, Beijing, China, 2018.

[24] M. Liu, The Influence of Traffic Restriction Measures on Air Quality in Lanzhou City Based on CMAQ, Lanzhou University, Lanzhou, China, 2018.
[25] Z. Liu, R. Li, X. Wang, and P. Shang, "Effects of vehicle restriction policies: analysis using license plate recognition data in Langfang, China," Transportation Research Part A: Policy and Practice, vol. 118, pp. 89-103, 2018.

[26] Y. Gu, E. Deakin, and Y. Long, "The effects of driving restrictions on travel behavior evidence from Beijing," Journal of Urban Economics, vol. 102, pp. 106-122, 2017.

[27] L. Wang, J. Xu, and P. Qin, "Will a driving restriction policy reduce car trips?-the case study of Beijing, China," Transportation Research Part A: Policy and Practice, vol. 67, pp. 279-290, 2014.

[28] V. Cantillo and J. De Dios Ortúzar, "Restricting the use of cars by license plate numbers: a misguided urban transport policy," Dyna, vol. 81, no. 188, pp. 75-82, 2014.

[29] L. de Grange and R. Troncoso, "Impacts of vehicle restrictions on urban transport flows: the case of Santiago, Chile," Transport Policy, vol. 18, no. 6, pp. 862-869, 2011.

[30] H. M. Worden, Y. Cheng, G. Pfister et al., "Satellite-based estimates of reduced $\mathrm{CO}$ and $\mathrm{CO}_{2}$ emissions due to traffic restrictions during the 2008 Beijing olympics," Geophysical Research Letters, vol. 39, no. 14, 2012.

[31] S. Chowdhury, S. Dey, S. N. Tripathi, G. Beig, A. K. Mishra, and S. Sharma, "“Traffic intervention" policy fails to mitigate air pollution in megacity Delhi," Environmental Science \& Policy, vol. 74, pp. 8-13, 2017.

[32] J. Ye, "Better safe than sorry? evidence from Lanzhou's driving restriction policy," China Economic Review, vol. 45, pp. 1-21, 2017.

[33] C. Sun, S. Zheng, and R. Wang, "Restricting driving for better traffic and clearer skies: did it work in Beijing?" Transport Policy, vol. 32, pp. 34-41, 2014.

[34] D. Han, H. Yang, and X. Wang, "Efficiency of the platenumber-based traffic rationing in general networks," Transportation Research Part E: Logistics and Transportation Review, vol. 46, no. 6, pp. 1095-1110, 2010.

[35] S. Wang, C. Shao, F. Wang, and J. Sun, "Evaluation on vehicle restriction measure in Beijing," in Proceedings of the Seventh International Conference on Traffic and Transportation Studies 2010, pp. 433-443, Kunming, China, August 2010.

[36] F. Gallego, J.-P. Montero, and C. Salas, "The effect of transport policies on car use: evidence from Latin American cities," Journal of Public Economics, vol. 107, pp. 47-62, 2013.

[37] H. Ma and G. He, "Effects of the post-olympics driving restrictions on air quality in Beijing," Sustainability, vol. 8, no. 9, p. 902, 2016.

[38] R. San Jose, J. L. Pérez, L. Pérez, and R. M. González, “A health impact assessment of traffic restrictions during Madrid $\mathrm{NO}_{2}$ episode," in Proceedings of the IOP Conference Series: 2018 9th International Conference on Environmental Science and Technology, IOP Publishing, Prague, Czech Republic, June 2018.

[39] D.-x. Wu, R. Li, and J.-f. Wu, "The economics analysis of the vehicle restriction rule," Technology \& Economy in Areas of Communications, vol. 2, 2012. 